\title{
Biochemical and Physiological Regulation of Cardiac Myocyte Contraction by Cardiac-Specific Myosin Light Chain Kinase
}

\author{
Osamu Tsukamoto, MD, PhD; Masafumi Kitakaze, $\mathrm{MD}, \mathrm{PhD}$
}

\begin{abstract}
Cardiac-specific myosin light chain kinase (cMLCK) is the kinase predominantly responsible for the maintenance of the basal level of phosphorylation of cardiac myosin light chain 2 (MLC2), which it phosphorylates at Ser-15. This phosphorylation repels the myosin heads from the thick myosin filament and moves them toward the thin actin filament. Unlike smooth muscle cells, MLC2 phosphorylation in striated muscle cells appears to be a positive modulator of $\mathrm{Ca}^{2+}$ sensitivity that shifts the $\mathrm{Ca}^{2+}$-force relationship toward the left and increases the maximal force response and thus does not initiate muscle contraction. Recent studies have revealed an increasing number of details of the biochemical, physiological, and pathophysiological characteristics of cMLCK. The combination of recent technological advances and the discovery of a novel class of biologically active nonstandard peptides will hopefully translate into the development of drugs for the treatment of heart diseases. (Circ $J$ 2013; 77: 2218-2225)
\end{abstract}

Key Words: $\mathrm{Ca}^{2+} /$ calmodulin; Muscle contraction; Myosin light chain kinase; Regulatory myosin light chain

A ctin and myosin are essential for the generation of contractile force. In 1954, Huxley and Hanson proposed the "sliding filament theory", which states that contractile force is generated through slippage of the cross-bridges between actin and myosin filaments ${ }^{1}$ and that purified actin and myosin are not sufficient for active movement. In 1962, intracellular calcium $\left(\left[\mathrm{Ca}^{2+}\right] \mathrm{i}\right)$ was identified as acting a trigger of muscle contraction. ${ }^{2}$ Although force generation can be achieved with only myosin and actin, additional proteins are required for its regulation. In smooth muscle, an increase in $\left[\mathrm{Ca}^{2+}\right] \mathrm{i}$ induces the binding of $\mathrm{Ca}^{2+} /$ calmodulin to smooth muscle-specific myosin light chain kinase (smMLCK), which leads to the phosphorylation of cardiac myosin light chain 2 (MLC2) in thick myosin filaments, activation of the ATPase of myosin heads and initiation of muscle contraction. ${ }^{3}$ Thus, smooth muscle cells use smMLCK as a $\left[\mathrm{Ca}^{2+}\right]$ i sensor and initiator of muscle contraction ${ }^{4}$ (Figure 1). In contrast, troponin is specifically expressed in skeletal and cardiac muscle cells and works as a sensing protein of $\left[\mathrm{Ca}^{2+}\right] \mathrm{i}^{5}{ }^{5}$ In contrast to smooth muscle cells, the initiation of striated muscle contraction is regulated primarily by the troponin-tropomyosin complex in thin actin filaments and not through MLC2 phosphorylation. The binding of $\mathrm{Ca}^{2+}$ to troponin $\mathrm{C}$ leads to relief of the inhibition of the binding of cross-bridges to thin actin filaments, and this relief triggers striated muscle contraction (Figure 1). However, endogenous expression of troponin proteins in smooth muscle cells was reported recently, ${ }^{6}$ although the physiological role of the troponin complex in the regulation of smooth muscle contraction still remains unknown.

Following the discovery of smMLCK and skeletal muscle MLCK (skMLCK), a third MLCK, named cardiac MLCK (cMLCK), was identified in $2007^{7}$ and is expressed exclusively in cardiac myocytes. However, what is the role of MLCK in striated muscle cells? Because both skeletal and cardiac muscle cells express specific MLCKs (ie, skMLCK and cMLCK, respectively), MLCKs might play specific physiological roles in each cell type.

\section{Intracellular Ca ${ }^{2+}$ Homeostasis in Muscle Tissues}

To set the stage for our discussion of MLCKs, it is necessary to understand the intracellular $\mathrm{Ca}^{2+}$ homeostasis in muscle tissues, because $\mathrm{Ca}^{2+}$ is a trigger of MLCK activation and the responses to $\left[\mathrm{Ca}^{2+}\right] \mathrm{i}$ differs among the 3 types of muscle cell: smooth muscle, skeletal muscle, and cardiac muscle (Figure 1). Local $\left[\mathrm{Ca}^{2+}\right] \mathrm{i}$ transients $\left(\mathrm{Ca}^{2+}\right.$ sparks $)$, which arise from the coordinated opening of a cluster of ryanodine-sensitive $\mathrm{Ca}^{2+}$ release channels, are necessary for effective $\mathrm{Ca}^{2+}$ signaling in all 3 types of muscle cell, although the rates of $\mathrm{Ca}^{2+}$ transients and contraction vary considerably in the different muscle cells. In general, however, these rates are slower in smooth muscle cells than in either skeletal or cardiac muscle cells. Smooth muscle cells gradually contract according to this slow increase in the level of $\left[\mathrm{Ca}^{2+}\right] \mathrm{i}^{8}{ }^{8}$ In skeletal muscle cells, the activation of the voltage-gated $\mathrm{Ca}^{2+}$ channel in the transverse tubule mem-

Received May 20, 2013; revised manuscript received June 7, 2013; accepted June 17, 2013; released online July 18, 2013

Department of Molecular Cardiology, Osaka University Graduate School of Medicine, Suita (O.T.); Department of Cardiovascular Medicine, National Cerebral and Cardiovascular Center, Suita (M.K.), Japan

Mailing address: Osamu Tsukamoto, MD, PhD, Department of Molecular Cardiology, Osaka University Graduate School of Medicine, 2-2

Yamadaoka, Suita 565-0871, Japan. E-mail: tsuka@medone.med.osaka-u.ac.jp

ISSN-1346-9843 doi:10.1253/circj.CJ-13-0627

All rights are reserved to the Japanese Circulation Society. For permissions, please e-mail: cj@j-circ.or.jp 


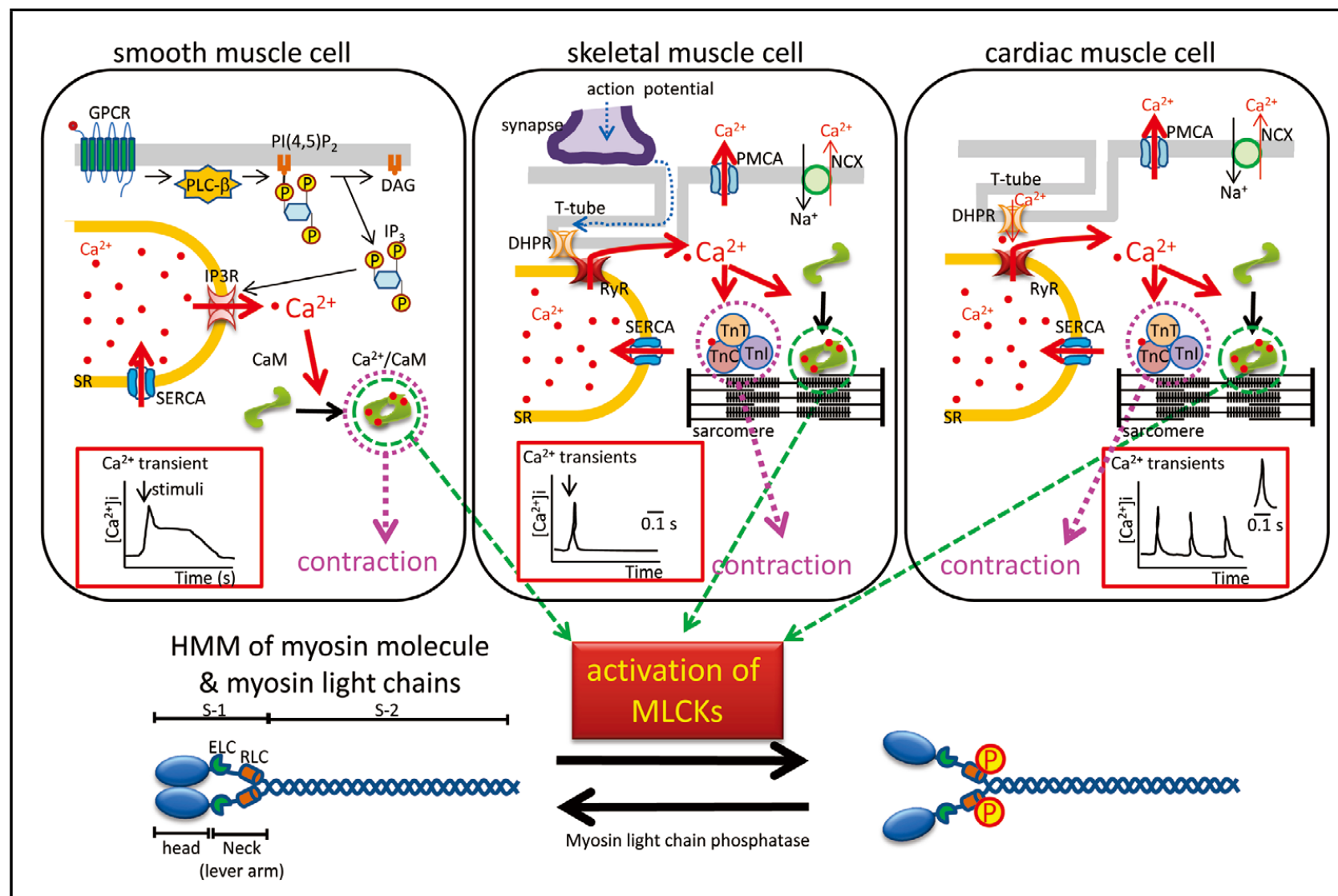

Figure 1. Intracellular calcium dynamics and muscle contraction. CaM, calmodulin; DAG, diacylglycerol; DHPR, dihydropyridine receptor; ELC, essential myosin light chain; GPCR, G protein-coupled receptor; IP3, inositol trisphosphate; IP3R, IP3 receptor; MLCK, myosin light chain kinase; NCX, sodium-calcium exchanger; PLC-b, phospholipase C beta; PI(4,5)P2, phosphatidylinositol 4,5-bisphosphate; PMCA, plasma membrane $\mathrm{Ca}^{2+}$-ATPase; SERCA, sarco/endoplasmic reticulum $\mathrm{Ca}^{2+}$-ATPase; SR, sarcoplasmic reticulum; RLC, regulatory myosin light chain; RyR, ryanodine receptor; TnC, troponin C; Tnl, troponin I; TnT, troponin T; T-tube, transverse tubules. For explanations, see text.

branes directly opens the ryanodine-sensitive $\mathrm{Ca}^{2+}$ release receptor on the sarcoplasmic reticulum (SR), which results in a rapid increase in $\left[\mathrm{Ca}^{2+}\right] \mathrm{i}$. In cardiac muscle cells, electrostimulation from the sinus node activates the voltage-dependent cation channel and thus increases the $\left[\mathrm{Ca}^{2+}\right] \mathrm{i}$ level, which induces a rapid and large release of $\mathrm{Ca}^{2+}$ from the $\mathrm{SR}$, known as " $\mathrm{Ca}^{2+}$-induced $\mathrm{Ca}^{2+}$ release". Importantly, the duration of the increased $\left[\mathrm{Ca}^{2+}\right] \mathrm{i}$ level in striated muscle cells is very short because the released $\mathrm{Ca}^{2+}$ is rapidly recaptured in the SR. In cardiac myocytes, electrostimulation increases $\left[\mathrm{Ca}^{2+}\right] \mathrm{i}$ from $100 \mathrm{nmol} / \mathrm{L}$ to a few hundred $\mathrm{nmol} / \mathrm{L}(\sim 1 \mu \mathrm{mol} / \mathrm{L})$, which returns to the basal level within $0.1 \mathrm{~s}$.

We now attempt to show why striated muscle cells do not use MLCK as a sensor of $\left[\mathrm{Ca}^{2+}\right] \mathrm{i}$ and an initiator of muscle contraction. The slow kinase reactions of MLCKs are not suitable as a $\left[\mathrm{Ca}^{2+}\right]$ i sensor in striated muscles, ${ }^{4}$ in which rapid and transient increase in the $\left[\mathrm{Ca}^{2+}\right] \mathrm{i}$ occurs. Instead, using a non-enzyme signal from the binding of $\mathrm{Ca}^{2+}$ to troponin, these cells contract promptly in response to the increased $\left[\mathrm{Ca}^{2+}\right] \mathrm{i}$. Thus, the increase in $\left[\mathrm{Ca}^{2+}\right] \mathrm{i}$ during muscle contraction exhibits distinct molecular kinetics in the 3 types of muscle, and these kinetics are related to the different physiological mechanisms that regulate contraction in these muscles.

\section{Mechanism of MLCK Activation by $\mathrm{Ca}^{2+} /$ Calmodulin Binding}

An increase in $\left[\mathrm{Ca}^{2+}\right]$ i can enhance MLCK activity approximately 1,000-fold through binding to calmodulin. $\mathrm{Ca}^{2+} /$ calmodulin is the most important regulator of MLCKs. MLCK is catalytically inactive in the absence of $\mathrm{Ca}^{2+} /$ calmodulin because the autoinhibitory sequence of MLCK blocks the access of the substrate to the catalytic core (Figure 2). ${ }^{9}$ The binding of $\mathrm{Ca}^{2+} /$ calmodulin to the calmodulin-binding domain displaces the autoinhibitory sequence from the surface of the catalytic core, which results in exposure of the catalytic site of the kinase and thus provides access to the N-terminus of MLC2.9 MLCK is maximally activated by $\mathrm{Ca}^{2+} /$ calmodulin at a molar ratio of $1: 1$ with a dissociation constant of $1 \mathrm{nmol} / \mathrm{L} .^{10}$

\section{Biochemistry of MLCKs}

\section{smMLCK}

smMLCK is encoded by the single-copy MYLK1 gene, which expresses 3 transcripts in a cell-specific manner related to alternative initiation sites: non-muscle isoform (longer form), smooth muscle isoform (shorter form), and telokin. ${ }^{11}$ Normally, smooth muscle expresses the shorter form of smMLCK. The smooth muscle isoform contains 3 DFRxxL motifs, a 


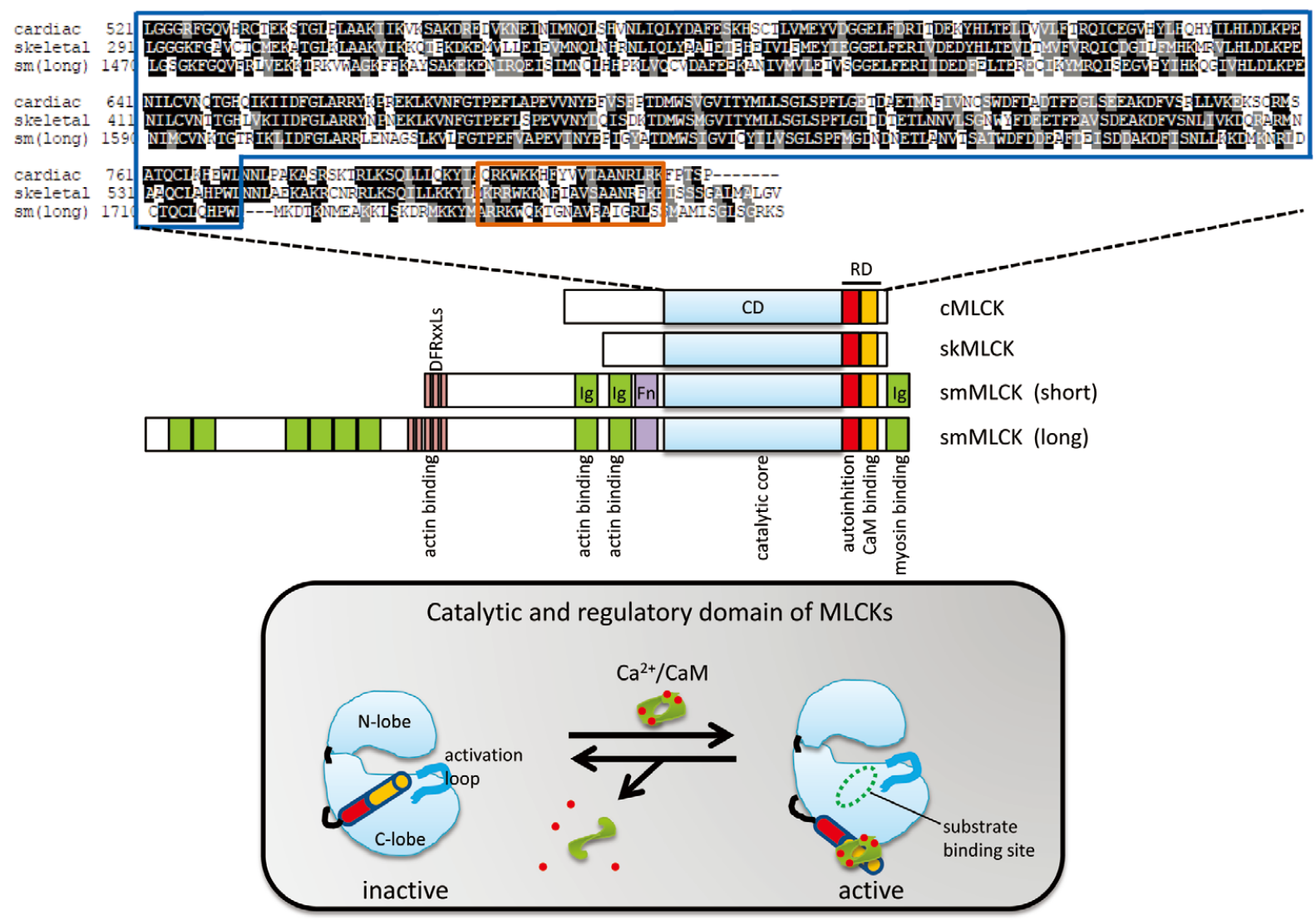

Figure 2. Structural and functional elements in myosin light chain kinase (MLCK) proteins. C, cardiac; CaM, calmodulin; CD catalytic domain; Fn, fibronectin domain; Ig, immunoglobulin domain; RD, regulatory domain; sk, skeletal; sm, smooth muscle;. For explanations, see text.

proline-rich repeat, 3 immunoglobulin (Ig) modules, 1 fibronectin (Fn) module, and 1 kinase domain with a catalytic core and a regulatory segment. The 3 DFRxxL motifs at the $\mathrm{N}$-terminus of smMLCK, which are not present in either of the striated muscle MLCKs, bind to thin actin filaments ${ }^{10}$ through an extension of the catalytic core toward the myosin thick filaments for the phosphorylation of the smooth muscle MLC2 at Ser-19 (Figure 2). ${ }^{12}$ smMLCK-induced Ser-19 phosphorylation activates myosin ATPase and initiates muscle contraction. ${ }^{3}$ Interestingly, different kinases are also known to phosphorylate smMLC2. Rho-associated coiled-coil forming kinase (ROCK) phosphorylates smMLC2 at Ser-19 to regulate the assembly of stress fibers. ${ }^{13}$ Protein kinase C (PKC) phosphorylates Ser-1/Ser-2/Thr-9, which inhibits myosin ATPase activity. ${ }^{14}$ In contrast, several protein kinases, including PKA, PKC, CaMKII, and PAK, are reported to phosphorylate serine residues in the calmodulin-binding sequence in the regulatory domain in vitro, which results in a 10-fold increase in $K_{\text {cam. }}{ }^{15}$ On the other hand, the phosphorylation of smMLCK at Thr-40 and Thr-43 by extracellular signal-regulated kinase (ERK) increases $V_{\max }$ without changing $K_{\mathrm{CaM}}{ }^{15} \mathrm{ATP}$, which is the other substrate of MLCK, can bind to the MLCK catalytic core regardless of the positioning of the autoinhibitory sequence. ${ }^{12}$ $K \mathrm{~m}$ for ATP is approximately $50-150 \mu \mathrm{mol} / \mathrm{L} .^{12}$ The concentrations of smMLCK and its substrate, smMLC2, are approximately $4 \mu \mathrm{mol} / \mathrm{L}$ and $30-40 \mu \mathrm{mol} / \mathrm{L}$, respectively. ${ }^{8}$

\section{skMLCK}

skMLCK is encoded by the MYLK2 gene and is predominantly expressed in skeletal muscle, although it was originally cloned from cardiac muscle. ${ }^{16}$ skMLCK is reported to weakly bind to myofilaments, ${ }^{10}$ likely because it lacks the actin binding domain that is found in smMLCK (Figure 2). skMLCK phosphorylates skeletal muscle MLC2 (skMLC2) at Ser-15. ${ }^{17}$ In contrast to smMLCK, which phosphorylates only smooth muscle MLC2 efficiently, skMLCK can phosphorylate other MLC2s found in cardiac and smooth muscles that exhibit similar catalytic properties. ${ }^{17}$ Previous physiological studies have demonstrated that the extent of MLC2 phosphorylation in skeletal muscle increases from $0-10 \%$ to $40-60 \%$ depending on the frequency of muscle stimulation. ${ }^{17}$

\section{CMLCK}

cMLCK is encoded by the MYLK3 gene and expressed exclusively in the heart, both the atria and ventricles. ${ }^{18}$ cMLCK is structurally related to both skMLCK and smMLCK and contains a conserved kinase domain at its $\mathrm{C}$-terminus that exhibits $58 \%$ identity with skMLCK and $44 \%$ identity with smMLCK. ${ }^{7,18}$ However, the N-terminus of cMLCK lacks homologies to known proteins, including other MLCKs, which indicates that cMLCK may play some specific functional roles (Figure 2). Immunostaining of endogenous cMLCK in cardiac myocytes has shown a diffuse positive staining pattern in 


\begin{tabular}{lccccc|}
\multicolumn{2}{l}{ Table. Biochemical Characteristics of Protein Kinases in the Heart } & & & \\
\multicolumn{1}{c}{ Kinase } & Gene & Substrate & $\boldsymbol{K}_{\mathbf{m}}(\boldsymbol{\mu} \mathbf{m o l} / \mathbf{L})$ & $\begin{array}{c}\boldsymbol{V}_{\mathbf{m a x}} \\
\left(\boldsymbol{\mu} \mathbf{m o l} \cdot \mathbf{m i n}^{-1} \cdot \mathbf{m g}^{-1}\right)\end{array}$ & $\boldsymbol{V}_{\text {max }} / \boldsymbol{K}_{\mathbf{m}}$ \\
Cardiac MLCK & MYLK3 & MLC2v & $4.3 \pm 1.5$ & $0.26 \pm 0.06$ & 0.06 \\
Skeletal MLCK & MYLK2 & Skeletal MLC2 & $4.3 \pm 0.5$ & $40 \pm 1.7$ & 9.3 \\
Smooth muscle MLCK & MYLK1 & Smooth muscle MLC2 & $8.3 \pm 1.4$ & $28 \pm 5.8$ & 3.5 \\
ZIPK & DAPK3 & MLC2v & $15.2 \pm 2.0$ & $0.89 \pm 0.05$ & 0.06 \\
& & Smooth muscle MLC2 & $1.8 \pm 0.3$ & $0.42 \pm 0.03$ & 0.23 \\
\hline
\end{tabular}

MLCK, myosin light chain kinase; MLC2v, ventricular myosin regulatory chain-2; ZIPK, zipper-interacting protein kinase.
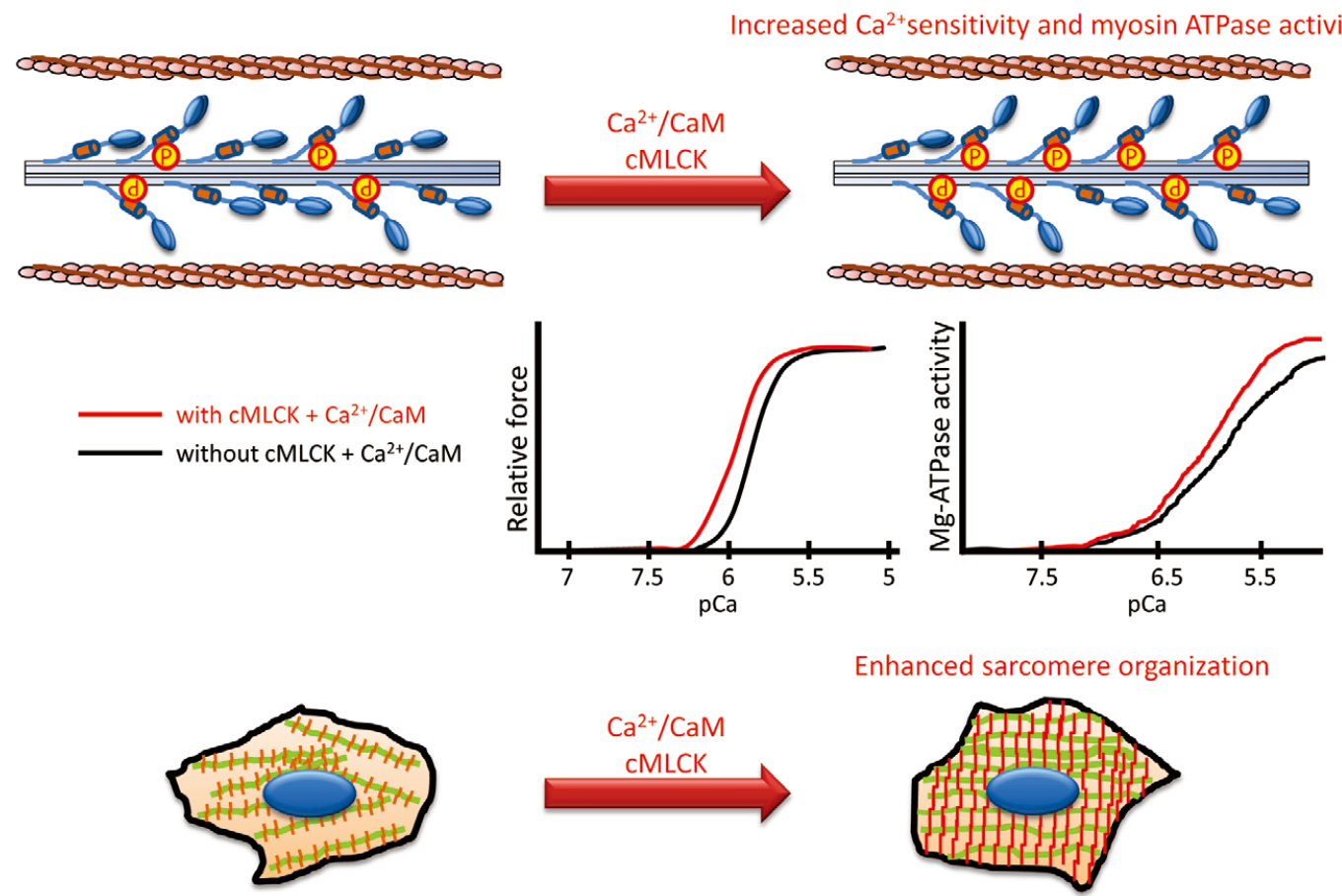

Enhanced sarcomere organization

Figure 3. Physiological and biochemical effects of cardiac myosin light chain 2 (MLC2) phosphorylation by cardiac myosin light chain kinase (cMLCK) on cardiac muscle contraction. cMLCK-induced MLC2 phosphorylation enhances the contraction of cardiac myocytes probably via a combination of increased $\mathrm{Ca}^{2+}$ sensitization, myosin Mg-ATPase activity, and sarcomere structural organization. CaM, calmodulin. For explanations, see text.

the cytoplasm with a striated staining pattern in the cell periphery. ${ }^{18}$ Interestingly, the striated MLCK staining colocalized with actin but not with its substrate. ${ }^{18}$ Chan et al reported the independence of cMLCK activity from $\mathrm{Ca}^{2+} /$ calmodulin, ${ }^{18}$ which was an unexpected result, because cMLCK also contains both autoinhibitory and calmodulin-binding sequences, similar to the other 2 types of MLCKs, and binds to calmodulin with high affinity in a $\mathrm{Ca}^{2+}$-dependent manner.,19 There are conflicting results concerning the $\mathrm{Ca}^{2+} /$ calmodulin dependency of cMLCK activity, ${ }^{7,19}$ and thus requires further examination.

The Table demonstrates the estimated kinetic constants of each MLCK determined by Lineweaver-Burk plots. ${ }^{18,20-22}$ cMLCK has a high affinity for its substrate, similar to skMLCK and smMLCK. However, the catalytic efficiency of cMLCK, which is indicated by the $V_{\mathrm{max}} / K_{\mathrm{m}}$ ratio, is lower than that of skMLCK and smMLCK. Thus, the maximal specific kinase activity of cMLCK is much lower than that of sm-
MLCK and skMLCK. However, the low specific activity of cMLCK results in a slow turnover of phosphate in MLC2 $(\mathrm{t} 1 / 2=250 \mathrm{~min})$, with an MLC2 basal phosphorylation of approximately $0.2-0.4 \mathrm{~mol}$ of phosphate/mol of MLC2 under basal conditions, ${ }^{23-26}$ which indicates that the kinase activity of cMLCK may be a primary limiting factor of MLC2 phosphorylation. ${ }^{19}$

\section{Structural Changes of Myosin Head Induced by MLC2 Phosphorylation in Striated Muscles}

Muscle myosin is the molecular motor in the thick filament of the sarcomere and is composed of 1 pair of myosin heavy chains (MHC) and 2 pairs of myosin light chains (MLC): essential MLC and regulatory MLC (ie, MLC2) (Figure 1). Both of the MLCs wrap around the neck region of the MHC. MLC2 is positioned at the $\mathrm{S} 1-\mathrm{S} 2$ junction of the MHC through its binding to a 35-amino-acid IQ motif on the MHC. ${ }^{27}$ The MLC2 
contains a highly conserved serine that is phosphorylatable by MLCK and plays an important role in the activation and modulation of myosin by fine-tuning the motion of the neck region of the MHC. ${ }^{10}$ The MLC2 also contains a $\mathrm{Ca}^{2+} / \mathrm{Mg}^{2+}$ binding site at its N-terminus from Asp-37 to Asp-48, located in the first helix-loop-helix motif, and the binding of divalent cation alters the structural and contractile properties. ${ }^{28,29}$ The neck region of the myosin head has been proposed to act as a lever arm. The phosphorylation of MLC2 at Ser-15 results in the addition of a negative charge to the $\mathrm{N}$-terminal region of MLC2, which induces the myosin head to swing out from a position close to the thick filament's backbone toward the actin filament, and this structural change increases the rate through which the myosin-actin interaction occurs and promotes force generation at a given level of $\mathrm{Ca}^{2+}\left(\right.$ Figures 1,3). ${ }^{30,31}$ Interestingly, several mutations around the phosphorylatable Ser-15 and the $\mathrm{Ca}^{2+}$ binding site in MLC2 have been found in patients with familial hypertrophic cardiomyopathy. ${ }^{32-34}$

\section{PKs and Protein Phosphatase Regulation of MLC2 Phosphorylation in the Heart}

There are 2 types of cardiac MLC2: a ventricular myosin light chain-2 (MLC2v) and an atrium-specific form (MLC2a). ${ }^{35}$ All 3 MLCKs are expressed in the heart. However, the amount of skMLCK in the heart is too low to maintain cardiac MLC2 phosphorylation, ${ }^{36}$ and ablation of skMLCK has no effect on MLC2 phosphorylation in cardiac muscle, ${ }^{36}$ which indicates that skMLCK does not play a major role in MLC2v phosphorylation. The expression of smMLCK in the heart is also 10- to 20-fold lower than that of cMLCK, ${ }^{18}$ and cardiac MLC2 is not a good substrate for smMLCK. ${ }^{36}$ smMLCK may phosphorylate non-muscle cytoplasmic myosin II-B and plays an important role in the heart. ${ }^{37}$ Thus, there are currently 2 candidate kinases for MLC2 phosphorylation in cardiac myocytes: $\mathrm{cMLCK}^{7,18}$ and zipper-interacting PK (ZIPK). ${ }^{38}$ ZIPK is a $\mathrm{Ca}^{2+}$-independent serine/threonine kinase that has been implicated in apoptosis and is ubiquitously expressed, including in adult and neonatal cardiac myocytes. ${ }^{39}$ In permeabilized smooth muscle cells, constitutively active ZIPK initiates contraction through the phosphorylation of smooth muscle MLC2. ${ }^{40}$ Cardiac MLC2 was also identified as a biochemically favorable substrate for ZIPK through an unbiased substrate search with purified ZIPK on heart homogenates and was phosphorylated at Ser-15 in vivo and in vitro by ZIPK. ${ }^{38}$ In fact, $V_{\max }$ for cardiac MLC2 is 2-fold greater than that obtained for smooth muscle MLC2 $\left(887 \pm 47\right.$ and $415 \pm 49 \mathrm{nmol} \cdot \mathrm{min}^{-1} \cdot \mathrm{mg}^{-1}$, respectively), whereas the $K_{\mathrm{m}}$ of cardiac MLC2 is higher than that of smooth muscle MLC2 $(15.0 \pm 2.0$ and $1.8 \pm 0.3 \mu \mathrm{mol} / \mathrm{L}$, respectively). ${ }^{38}$ However, ZIPK is considered not to be involved in the basal phosphorylation of cardiac MLC2 in vivo, ${ }^{41}$ although knockdown of ZIPK in cardiac myocytes by siRNA decreased the extent of cardiac regulatory MLC phosphorylation by $34 \% .{ }^{38}$ The physiological role of ZIPK-induced cardiac MLC2 phosphorylation remains unknown.

It is now well established that cMLCK is the predominant cardiac MLC2 kinase responsible for basal phosphorylation in vivo, because the ablation of cMLCK almost completely abolishes the phosphorylation of MLC2v..$^{7,18,25,41}$ Ding et al demonstrated that a partial reduction in the amount of cMLCK protein in cMLCK hetero knockout mice $\left(\mathrm{cMLCK}^{+/ n e o}\right)$ resulted in a partial reduction in MLC2 phosphorylation in both ventricular and atrial muscles, and this reduction in MLC2 phosphorylation was proportional to the cMLCK expression level. ${ }^{41}$ In addition, the overexpression of cMLCK increases MLC2v phosphorylation in a dose-dependent manner and, conversely, knockdown of cMLCK by RNAi decreases MCL2 $v$ phosphorylation in vitro. ${ }^{18}$ Scruggs et al identified 3 distinct charge variants of endogenous MLC2 $v$ in vivo in the mouse: unphosphorylated, singly phosphorylated, and doubly phosphorylated at Ser-14/Ser-15.42 In contrast, Ser-14 in murine MLC2v is replaced by Asn in human MLC2. However, human MLC2 also has 3 distinct charge variants in vivo: unphosphorylated, singly phosphorylated at Ser-15, and deamidated Asn-14/ phosphorylated Ser-15. ${ }^{42}$ Interestingly, the deamination of Asn to Asp can create a negative charge similar to that obtained through phosphorylation. ${ }^{26}$ In addition, there is a spatial gradient of MLC2v phosphorylation through the ventricular wall: relatively low in the inner layer and high in the outer layer. ${ }^{16,43}$ This gradient, which may be caused by reduced activity of the phosphatase in the outer layer, ${ }^{44,45}$ may be important for normalizing wall stress and contributes to efficient contraction of the whole heart. ${ }^{16}$

The level of MLC2 $\mathrm{v}$ phosphorylation is maintained relatively constant by the appropriate balance between phosphorylation by cMLCK and dephosphorylation by phosphatase in the physiologically constant beating heart. The dephosphorylation of MLC2v is mediated mainly by catalytic subunit of type 1 phosphatase (PP1c- $\delta$ ) in concert with myosin phosphatase target subunit 2 (MYPT2). ${ }^{46}$ In contrast, MLC2 phosphorylation by MLCK is transient in both smooth and skeletal muscle cells, although they have different MLC2 phosphorylation dynamics. ${ }^{19}$ MLC2 phosphorylation is rapidly dephosphorylated by robust myosin light chain phosphatase activity in smooth muscle cells, whereas MLC2 phosphorylation is prolonged and slowly dephosphorylated by the low myosin light chain phosphatase activity in skeletal muscle cells.

\section{Physiological Regulators of cMLCK Expression and Activity}

The expression of cMLCK is highly regulated by the cardiac homeobox protein Nkx2-5 in neonatal cardiac myocytes. ${ }^{18}$ cMLCK mRNA increases during development to adult stages and persists in the aged heart, whereas its protein level decreases in the aged heart, which suggests an alternation in post-transcriptional regulation. ${ }^{18}$ Exercise has been reported to increase cMLCK expression and MLC2v phosphorylation. ${ }^{25}$ Catalytic activity may be regulated by phosphorylation through upstream kinases. ${ }^{47} \mathrm{cMLCK}$ has several potential phosphorylation sites for other kinases, such as PKA and PKC, ${ }^{18}$ although more studies are required to clarify the details of this mechanism.

Hypertrophic agonists, such as $\alpha 1$ - or $\beta 1$-adrenergic stimulation ${ }^{18,48,49}$ and angiotensin II, induce MLC2 $\mathrm{v}$ phosphorylation through MLCK activation in both cultured cardiac myocytes and the adult heart in vivo. ${ }^{24}$ Other neurohumoral stimulators, such as endothelin ${ }^{50}$ and prostanoid $\mathrm{F}$ receptors, ${ }^{51}$ have also been reported to increase MLC2 phosphorylation and induce a prominent increase in the contractile force in the heart. Neuregulin, which activates ErbB receptor tyrosine kinase, has been reported to enhance the expression of cMLCK with a concomitant increase in MLC2 $\mathrm{v}$ phosphorylation and improved cardiac performance after myocardial infarction in rats. ${ }^{52}$

\section{Role of MLC2 Phosphorylation in Sarcomere Organization and Heart Development}

The transfection of cardiac myocytes with skMLCK has resulted in the phosphorylation of MLC2 $\mathrm{v}$ and led to a highly 
organized sarcomeric pattern without induction of other hypertrophic phenotypes, such as the induction of fetal genes and an increase in cell size. ${ }^{24}$ Furthermore, the dominant-negative kinase-inactive form of MLCK completely prevents sarcomere organization in response to angiotensin II ${ }^{24}$ which suggests that MLCK activation is necessary and sufficient to induce sarcomere organization (Figure 3). Consistent with these findings, the adenovirus-mediated overexpression of cMLCK in cardiac myocytes promotes sarcomere organization characterized by straight, thick, striated actin bundles, ${ }^{18}$ whereas reestablishment of the phenylephrine-induced sarcomere structure is inhibited by pretreatment with RNAi against cMLCK. ${ }^{7}$ Interestingly, the reduced cMLCK expression induced by the antisense morpholino causes severely impaired heart development in zebrafish and histological analysis showed that the structure of the sarcomere was poorly developed compared with control zebrafish. ${ }^{7}$ However, studies with transgenic and knockout mice have shown that MLC2 $\mathrm{v}$ phosphorylation is not critical for cardiogenesis in the mammalian system, ${ }^{18,41,53}$ although it is necessary for the optimal contractile performance of the heart.

\section{Physiological Role of MLC2 Phosphorylation in the Heart}

The degree of MLC2 phosphorylation is known to play a critical role in the determination of the $\mathrm{Ca}^{2+}$-sensitive cross-bridge transition in skeletal muscle. ${ }^{54}$ In permeable cardiac muscle fibers, MLC2 phosphorylation induced by MLCK increases the $\mathrm{Ca}^{2+}$ sensitivity, which manifests as a leftward shift in the force- $\mathrm{Ca}^{2+}$ relationship, and MLC2 dephosphorylation by phosphatase decreases the $\mathrm{Ca}^{2+}$ sensitivity (ie, dephosphorylation resulted in $\mathrm{Ca}^{2+}$ desensitization). ${ }^{55,56}$ MLC2 phosphorylation by MLCK is also associated with enhanced $\mathrm{Ca}^{2+}$-stimulated myosin Mg-ATPase activity in rat cardiac myofibrils (Figure 3) ${ }^{57}$ In the rat heart, MLC2v phosphorylation increases in response to an increase in the beat frequency and/or left ventricular pressure by exercise or inotropic agents, which may help augment the peak left ventricular pressure. ${ }^{58,59}$ At the cellular level, adenovirus-mediated overexpression of cMLCK potentiates the amplitude of the contraction of cardiac myocytes and the kinetics of contraction and relaxation without changing the $\left[\mathrm{Ca}^{2+}\right] \mathrm{i}$ transients. ${ }^{18}$

Several studies have also addressed the role of MLC2 phosphorylation in the heart using genetically modified mice. Huang et $\mathrm{al}^{60}$ generated transgenic mice expressing skMLCK specifically in cardiac myocytes (TG-skMLCK). These TGskMLCK mice demonstrated marked increases in the phosphorylation of both cardiac MLC2 and cytoplasmic non-muscle MLC2 in the heart without significant cardiac hypertrophy or structural abnormalities up to 6 months of age, which indicates that increased cardiac MLC2 phosphorylation per se does not cause cardiac hypertrophy. ${ }^{60}$ Interestingly, the hypertrophic cardiac response to exercise and isoproterenol treatment was attenuated in TG-skMLCK mice, ${ }^{26}$ which supports the hypothesis that the phosphorylation of cardiac MLC2 may inhibit physiological and pathophysiological hypertrophic responses through enhanced contractile performance and efficiency. Sanbe et $\mathrm{al}^{53}$ created transgenic mice (TG-MLC2v(P-)) in which 3 potentially phosphorylatable serines (Ser-14/Ser$15 /$ Ser-19) in the MLC2v (ventricular regulatory myosin light chain) were mutated to alanine. After MLCK treatment, the isolated ventricular fibers from the non-transgenic control mice showed increased $\mathrm{Mg}$-ATPase activity and $\mathrm{Ca}^{2+}$ sensitivity, as indicated by a leftward shift in the force- $\mathrm{Ca}^{2+}$ curve, whereas the fibers from the TG-MLC2v(P-) mice did not exhibit these increases. ${ }^{53}$ This suppressed performance of muscle fibers with nonphosphorable MLC2v is consistent with the demonstrated effects of MLC2 phosphorylation in skeletal muscle. ${ }^{36}$ Scruggs et al $^{48}$ examined the role of regulatory myosin light chain 2 phosphorylation in the ejection of the hearts of TG-MLC2v(P-) mice by measuring the systolic mechanics under basal conditions and in response to adrenergic stimulation. The TG-MLC2v(P-) mice demonstrated depressed contractility, decreased maximal left ventricular power development, and a decrease in the time-to-peak elastance during ejection under basal conditions. ${ }^{48}$ Interestingly, the TGMLC2v(P-) mice exhibited a blunting of the positive inotropic response to $\beta 1$-adrenergic stimulation. ${ }^{48}$ Because $\mathrm{cMLCK}$ has multiple PKA consensus sequences in its unique $\mathrm{N}$-terminus region, ${ }^{26}$ and $\beta 1$-adrenergic stimulation increased MLC2v Ser15 phosphorylation in hearts of non-transgenic control mice ${ }^{48}$, there might be a possible relationship between $\beta 1$-adrenergic signaling and MLC2v phosphorylation. The TG-MLC2v(P-) mice developed cardiac hypertrophy at 3-4 months of age, most likely because of a compensatory hypertrophic growth response to diminished contractile performance. ${ }^{53} \mathrm{cMLCK}$ knockout mice (cMLCK ${ }^{\text {neo/neo }}$ ) also demonstrated the critical role of cMLCK in normal physiological cardiac function, with decreased cardiac performance and the induction of cardiac hypertrophy at 4-5 months of age. ${ }^{41}$ In contrast, transgenic mice overexpressing MYPT2 specifically in cardiac myocytes demonstrated enhanced expression of MYPT2 with a concomitant increase in the level of endogenous PP1c- $\delta$, which resulted in a reduction of the level of in vivo MLC2 $v$ phosphorylation in the heart associated with a decrease in the myofilament response to $\mathrm{Ca}^{2+}$ and a decreased left ventricular contractility. ${ }^{61}$ These findings are consistent with the evidence obtained from TG-MLC2v(P-) mice and cMLCK-knockout mice as mentioned earlier.

Surprisingly, Warren et al recently reported an inverse relationship between cMLCK expression and systolic pressure: cMLCK expression is higher in the right ventricular myocardium than the left ventricular myocardium. ${ }^{25}$ Because the cMLCK expression in the left ventricle was markedly downregulated as a result of pressure overload, those authors speculated that increased mechanical stress reduces the net expression of cMLCK. ${ }^{25}$

\section{cMLCK in Heart Diseases}

cMLCK was first identified through the integrated cDNA expression analysis of failing human myocardium, which showed that the cMLCK mRNA expression levels correlated well with the pulmonary arterial pressure of patients with heart failure. ${ }^{7}$ Decreased phosphorylation of MLC2 was also reported in some patients with heart failure. ${ }^{62,63}$ In animal models of myocardial infarction, the expression level of cMLCK in the heart has been found to be reduced. ${ }^{18,52}$ In addition, pressure overload also led to a marked reduction in cMLCK and phosphorylated MLC2v in the heart 1 week after thoracic aortic constriction surgery. ${ }^{25}$ Interestingly, the reduction in the cMLCK protein level in pressure-overloaded hearts was mediated by upregulation of the ubiquitin-proteasome degradation system. ${ }^{25}$ The specific overexpression of cMLCK in cardiac myocytes attenuated the phenotype of the pressure overload-induced heart failure, ${ }^{25}$ and this finding suggests a protective role of cMLCK against cardiac stress.

Induced pluripotent stem cell-derived cardiac myocytes (iPSC-CMs) are expected to become a new cell therapy for 
heart diseases and iPSC-CMs express cardiac-specific proteins similar to neonatal cardiac myocytes. ${ }^{64}$ However, so far there are no reports of investigations into cMLCK and MLC2 phosphorylation in iPSC-CMs.

\section{New Approach to Finding Potential Inhibitors or Activators of Kinases}

Recently, Suga et al developed a new technology to discover "natural product-like" nonstandard peptides against various therapeutic targets, and this technology, which is known as the RaPID (Random non-strand Peptides Integrated Discovery) system, comprises a FIT (Flexible in vivo Translation) system coupled with an mRNA display. ${ }^{65}$ Using this system, Suga et al successfully isolated anti-E6AP macrocyclic $N$-methyl-peptides, one of which had high affinity $\left(K_{\mathrm{d}}=0.60 \mathrm{nmol} / \mathrm{L}\right)$ against a target E6AP and strongly suppressed its polyubiquitination ability against p53. ${ }^{66}$ Because the targets of the RaPID system are not limited, this technology can be applied to the discovery of kinase activators or inhibitors.

\section{Conclusion}

We are now attempting to find a new potential activator or inhibitor of cMLCK, which will hopefully either provide new insights to cMLCK or be successfully applied in the clinical setting.

\section{Acknowledgments}

This research was supported by the Japan Society for the Promotion of Science (JSPS) through the "Funding Program for Next Generation WorldLeading Researchers (NEXT Program)," initiated by the Council for Science and Technology Policy (CSTP); grants-in-aid from the Ministry of Health, Labor, and Welfare-Japan; grants-in-aid from the Ministry of Education, Culture, Sports, Science, and Technology-Japan. The research was also supported by grants from the Japan Heart Foundation, Japan Cardiovascular Research Foundation, Japan Cardiovascular Research Foundation, Japan Foundation of Applied Enzymology.

Conflict of Interest: The authors have no conflicts of interest to declare.

\section{References}

1. Huxley H, Hanson J. Changes in the cross-striations of muscle during contraction and stretch and their structural interpretation.1954.

2. Ebashi F, Ebashi S. Removal of calcium and relaxation in actomyosin systems. Nature 1962; 194: 378-379.

3. Kamm KE, Stull JT. The function of myosin and myosin light chain kinase phosphorylation in smooth muscle. Annu Rev Pharmacol Toxicol 1985; 25: 593-620.

4. Koizumi K, Hoshiai M, Ishida H, Ohyama K, Sugiyama H, Naito A, et al. Stanniocalcin 1 prevents cytosolic $\mathrm{Ca}^{2+}$ overload and cell hypercontracture in cardiomyocytes. Circ J 2007; 71: 796-801.

5. Ebashi S, Kodama A. A new protein factor promoting aggregation of tropomyosin. J Biochem 1965; 58: 107-108.

6. Kajioka S, Takahashi-Yanaga F, Shahab N, Onimaru M, Matsuda M, Takahashi R, et al. Endogenous cardiac troponin T modulates $\mathrm{Ca}(2+)-$ mediated smooth muscle contraction. Sci Rep 2012; 2: 979

7. Seguchi O, Takashima S, Yamazaki S, Asakura M, Asano Y, Shintani $\mathrm{Y}$, et al. A cardiac myosin light chain kinase regulates sarcomere assembly in the vertebrate heart. J Clin Invest 2007; 117: 2812-2824.

8. Takashima S. Phosphorylation of myosin regulatory light chain by myosin light chain kinase, and muscle contraction. Circ J 2009; 73: $208-213$.

9. Chin D, Means AR. Calmodulin: A prototypical calcium sensor. Trends Cell Biol 2000; 10: 322-328.

10. Sweeney HL, Bowman BF, Stull JT. Myosin light chain phosphorylation in vertebrate striated muscle: Regulation and function. Am J Physiol 1993; 264: C1085-C1095.

11. Birukov KG, Schavocky JP, Shirinsky VP, Chibalina MV, Van Eldik LJ, Watterson DM. Organization of the genetic locus for chicken myosin light chain kinase is complex: Multiple proteins are encoded and exhibit differential expression and localization. J Cell Biochem 1998; 70: 402-413.
12. Hong F, Haldeman BD, Jackson D, Carter M, Baker JE, Cremo CR. Biochemistry of smooth muscle myosin light chain kinase. Arch Biochem Biophys 2011; 510: 135-146.

13. Totsukawa G, Yamakita Y, Yamashiro S, Hartshorne DJ, Sasaki Y, Matsumura F. Distinct roles of ROCK (Rho-kinase) and MLCK in spatial regulation of MLC phosphorylation for assembly of stress fibers and focal adhesions in $3 \mathrm{~T} 3$ fibroblasts. J Cell Biol 2000; 150: 797-806.

14. Ikebe M, Hartshorne DJ, Elzinga M. Phosphorylation of the 20,000dalton light chain of smooth muscle myosin by the calcium-activated, phospholipid-dependent protein kinase: Phosphorylation sites and effects of phosphorylation. J Biol Chem 1987; 262: 9569-9573.

15. Kamm KE, Stull JT. Dedicated myosin light chain kinases with diverse cellular functions. J Biol Chem 2001; 276: 4527-4530.

16. Davis JS, Hassanzadeh S, Winitsky S, Lin H, Satorius C, Vemuri R, et al. The overall pattern of cardiac contraction depends on a spatial gradient of myosin regulatory light chain phosphorylation. Cell 2001; 107: $631-641$.

17. Stull JT, Kamm KE, Vandenboom R. Myosin light chain kinase and the role of myosin light chain phosphorylation in skeletal muscle. Arch Biochem Biophys 2011; 510: 120-128.

18. Chan JY, Takeda M, Briggs LE, Graham ML, Lu JT, Horikoshi N, et al. Identification of cardiac-specific myosin light chain kinase. Circ Res 2008; 102: 571-580.

19. Kamm KE, Stull JT. Signaling to myosin regulatory light chain in sarcomeres. J Biol Chem 2011; 286: 9941-9947.

20. Zhi G, Herring BP, Stull JT. Structural requirements for phosphorylation of myosin regulatory light chain from smooth muscle. J Biol Chem 1994; 269: 24723-24727.

21. Herring BP, Gallagher PJ, Stull JT. Substrate specificity of myosin light chain kinases. J Biol Chem 1992; 267: 25945-25950.

22. Ikebe M, Reardon S, Schwonek JP, Sanders CR 2nd, Ikebe R. Structural requirement of the regulatory light chain of smooth muscle myosin as a substrate for myosin light chain kinase. J Biol Chem 1994; 269: $28165-28172$.

23. Herring BP, England PJ. The turnover of phosphate bound to myosin light chain-2 in perfused rat heart. Biochem J 1986; 240: 205-214.

24. Aoki H, Sadoshima J, Izumo S. Myosin light chain kinase mediates sarcomere organization during cardiac hypertrophy in vitro. Nat Med 2000; 6: $183-188$.

25. Warren SA, Briggs LE, Zeng H, Chuang J, Chang EI, Terada R, et al. Myosin light chain phosphorylation is critical for adaptation to cardiac stress. Circulation 2012; 126: 2575-2588.

26. Scruggs SB, Solaro RJ. The significance of regulatory light chain phosphorylation in cardiac physiology. Arch Biochem Biophys 2011; 510: $129-134$.

27. Rayment I, Rypniewski WR, Schmidt-Base K, Smith R, Tomchick DR, Benning MM, et al. Three-dimensional structure of myosin subfragment-1: A molecular motor. Science 1993; 261: 50-58.

28. Reinach FC, Nagai K, Kendrick-Jones J. Site-directed mutagenesis of the regulatory light-chain $\mathrm{Ca}^{2+} / \mathrm{Mg}^{2+}$ binding site and its role in hybrid myosins. Nature 1986; 322: 80-83.

29. Szczesna-Cordary D, Guzman G, Ng SS, Zhao J. Familial hypertrophic cardiomyopathy-linked alterations in $\mathrm{Ca}^{2+}$ binding of human cardiac myosin regulatory light chain affect cardiac muscle contraction. J Biol Chem 2004; 279: 3535-3542.

30. Levine RJ, Chantler PD, Kensler RW, Woodhead JL. Effects of phosphorylation by myosin light chain kinase on the structure of Limulus thick filaments. J Cell Biol 1991; 113: 563-572.

31. Levine RJ, Kensler RW, Yang Z, Stull JT, Sweeney HL. Myosin light chain phosphorylation affects the structure of rabbit skeletal muscle thick filaments. Biophys J 1996; 71: 898-907.

32. Szczesna D, Ghosh D, Li Q, Gomes AV, Guzman G, Arana C, et al. Familial hypertrophic cardiomyopathy mutations in the regulatory light chains of myosin affect their structure, $\mathrm{Ca}^{2+}$ binding, and phosphorylation. J Biol Chem 2001; 276: 7086-7092.

33. Otsuka H, Arimura T, Abe T, Kaiwai H, Aizawa Y, Kubo T, et al. Prevalence and distribution of sarcomeric gene mutations in Japanese patients with familial hypertrophic cardiomyopathy. Circ J 2012; 76: $453-461$.

34. Niwano S. Multicenter study of the prevalence and distribution of sarcometric gene mutations in familial hypertrophic cardiomyopathy: A milestone for genetic diagnosis in the Japanese population. Circ J 2012; 76: 303-304.

35. Collins JH. Myoinformatics report: Myosin regulatory light chain paralogs in the human genome. J Muscle Res Cell Motil 2006; 27: 69-74.

36. Zhi G, Ryder JW, Huang J, Ding P, Chen Y, Zhao Y, et al. Myosin light chain kinase and myosin phosphorylation effect frequencydependent potentiation of skeletal muscle contraction. Proc Natl 
Acad Sci USA 2005; 102: 17519-17524.

37. Ma X, Takeda K, Singh A, Yu ZX, Zerfas P, Blount A, et al. Conditional ablation of nonmuscle myosin II-B delineates heart defects in adult mice. Circ Res 2009; 105: 1102-1109.

38. Chang AN, Chen G, Gerard RD, Kamm KE, Stull JT. Cardiac myo$\sin$ is a substrate for zipper-interacting protein kinase (ZIPK). J Biol Chem 2010; 285: 5122-5126.

39. Haystead TA. ZIP kinase, a key regulator of myosin protein phosphatase 1. Cell Signal 2005; 17: 1313-1322.

40. Ihara E, MacDonald JA. The regulation of smooth muscle contractility by zipper-interacting protein kinase. Can J Physiol Pharmacol 2007; 85: 79-87.

41. Ding P, Huang J, Battiprolu PK, Hill JA, Kamm KE, Stull JT. Cardiac myosin light chain kinase is necessary for myosin regulatory light chain phosphorylation and cardiac performance in vivo. $J$ Biol Chem 2010; 285: 40819-40829.

42. Scruggs SB, Reisdorph R, Armstrong ML, Warren CM, Reisdorph $\mathrm{N}$, Solaro RJ, et al. A novel, in-solution separation of endogenous cardiac sarcomeric proteins and identification of distinct charged variants of regulatory light chain. Mol Cell Proteomics 2010; 9: $1804-1818$

43. Hidalgo C, Wu Y, Peng J, Siems WF, Campbell KB, Granzier H. Effect of diastolic pressure on MLC2 $v$ phosphorylation in the rat left ventricle. Arch Biochem Biophys 2006; 456: 216-223.

44. Rajashree R, Blunt BC, Hofmann PA. Modulation of myosin phosphatase targeting subunit and protein phosphatase 1 in the heart. $\mathrm{Am}$ J Physiol Heart Circ Physiol 2005; 289: H1736-H1743.

45. Cohen PT. Protein phosphatase 1: Targeted in many directions. $J$ Cell Sci 2002; 115: 241-256.

46. Fujioka M, Takahashi N, Odai H, Araki S, Ichikawa K, Feng J, et al. A new isoform of human myosin phosphatase targeting/regulatory subunit (MYPT2): cDNA cloning, tissue expression, and chromosomal mapping. Genomics 1998; 49: 59-68.

47. Soderling TR, Stull JT. Structure and regulation of calcium/calmodulin-dependent protein kinases. Chem Rev 2001; 101: 2341-2352.

48. Scruggs SB, Hinken AC, Thawornkaiwong A, Robbins J, Walker $\mathrm{LA}$, de Tombe PP, et al. Ablation of ventricular myosin regulatory light chain phosphorylation in mice causes cardiac dysfunction in situ and affects neighboring myofilament protein phosphorylation. J Biol Chem 2009; 284: 5097-5106.

49. Grimm M, Haas P, Willipinski-Stapelfeldt B, Zimmerman WH, Rau $\mathrm{T}$, Pantel K, et al. Key role of myosin light chain (MLC) kinasemediated MLC2a phosphorylation in the alpha 1-adrenergic positive inotropic effect in human atrium. Cardiovasc Res 2005; 65: 211 220.

50. Rossmanith GH, Hoh JF, Turnbull L, Ludowyke RI. Mechanism of action of endothelin in rat cardiac muscle: Cross-bridge kinetics and myosin light chain phosphorylation. J Physiol 1997; 505: 217-227.

51. Riise J, Nguyen CH, Qvigstad E, Sandnes DL, Osnes JB, Skomedal $\mathrm{T}$, et al. Prostanoid $\mathrm{F}$ receptors elicit an inotropic effect in rat left ventricle by enhancing myosin light chain phosphorylation. Cardiovasc Res 2008; 80: 407-415.
52. Gu X, Liu X, Xu D, Li X, Yan M, Qi Y, et al. Cardiac functional improvement in rats with myocardial infarction by up-regulating cardiac myosin light chain kinase with neuregulin. Cardiovasc Res 2010; 88: $334-343$.

53. Sanbe A, Fewell JG, Gulick J, Osinska H, Lorenz J, Hall DG, et al. Abnormal cardiac structure and function in mice expressing nonphosphorylatable cardiac regulatory myosin light chain 2 . J Biol Chem 1999; 274: 21085-21094.

54. Patel JR, Diffee GM, Moss RL. Myosin regulatory light chain modulates the $\mathrm{Ca}^{2+}$ dependence of the kinetics of tension development in skeletal muscle fibers. Biophys J 1996; 70: 2333-2340.

55. Morano I, Hofmann F, Zimmer M, Ruegg JC. The influence of Plight chain phosphorylation by myosin light chain kinase on the calcium sensitivity of chemically skinned heart fibres. FEBS Lett 1985; 189: 221-224.

56. Sweeney HL, Stull JT. Phosphorylation of myosin in permeabilized mammalian cardiac and skeletal muscle cells. Am J Physiol 1986; 250: C657-C660.

57. Noland TA Jr, Kuo JF. Phosphorylation of cardiac myosin light chain 2 by protein kinase $\mathrm{C}$ and myosin light chain kinase increases $\mathrm{Ca}(2+)$-stimulated actomyosin MgATPase activity. Biochem Biophys Res Commun 1993; 193: 254-260.

58. Fitzsimons DP, Bodell PW, Baldwin KM. Phosphorylation of rodent cardiac myosin light chain 2: Effects of exercise. J Appl Physiol 1989; 67: 2447-2453.

59. Fitzsimons DP, Bodell PW, Baldwin KM. Myocardial functional correlates of cardiac myosin light chain 2 phosphorylation. $J$ Appl Physiol 1990; 68: 2426-2433.

60. Huang J, Shelton JM, Richardson JA, Kamm KE, Stull JT. Myosin regulatory light chain phosphorylation attenuates cardiac hypertrophy. J Biol Chem 2008; 283: 19748-19756.

61. Mizutani H, Okamoto R, Moriki N, Konishi K, Taniguchi M, Fujita $\mathrm{S}$, et al. Overexpression of myosin phosphatase reduces $\mathrm{Ca}(2+)$ sensitivity of contraction and impairs cardiac function. Circ J 2010; 74: $120-128$.

62. Morano I. Effects of different expression and posttranslational modifications of myosin light chains on contractility of skinned human cardiac fibers. Basic Res Cardiol 1992; 87(Suppl 1): 129-141.

63. van der Velden J, Papp Z, Boontje NM, Zaremba R, de Jong JW, Janssen PM, et al. The effect of myosin light chain 2 dephosphorylation on $\mathrm{Ca}^{2+}$-sensitivity of force is enhanced in failing human hearts. Cardiovasc Res 2003; 57: 505-514.

64. Yu T, Miyagawa S, Miki K, Saito A, Fukushima S, Higuchi T, et al. In vivo differentiation of induced pluripotent stem cell-derived cardiomyocytes. Circ J 2013; 77: 1297-1306.

65. Hipolito CJ, Suga H. Ribosomal production and in vitro selection of natural product-like peptidomimetics: The FIT and RaPID systems. Curr Opin Chem Biol 2012; 16: 196-203.

66. Yamagishi Y, Shoji I, Miyagawa S, Kawakami T, Katoh T, Goto Y, et al. Natural product-like macrocyclic N-methyl-peptide inhibitors against a ubiquitin ligase uncovered from a ribosome-expressed de novo library. Chem Biol 2011; 18: 1562-1570. 